\title{
Correction to: Docking and in silico toxicity assessment of Arthrospira compounds as potential antiviral agents against SARS-CoV-2
}

\author{
Léna Petit ${ }^{1}$ (D) - Léa Vernès ${ }^{1}$ (D) - Jean-Paul Cadoret ${ }^{1}$ (D) \\ Published online: 10 May 2021 \\ (C) Springer Nature B.V. 2021
}

\section{Correction to: J Appl Phycol} https://doi.org/10.1007/s10811-021-02372-9

Table 6 in the original version of this article contained an error. The corrected table and table legend are presented below.

$\triangle$ Jean-Paul Cadoret jean.paul.cadoret@algamafoods.com

1 Algama, 81 rue Réaumur, 75002 Paris, France 
Table 6 Docking results for small molecules vs. Spike RBD of SARS-CoV-2

\begin{tabular}{|c|c|c|c|c|c|c|c|}
\hline \multirow{3}{*}{$\begin{array}{c}\text { Docking softwares } \\
\text { Molecules }\end{array}$} & \multicolumn{6}{|c|}{ Binding energies (kcal.mol ${ }^{-1}$ ) } & \multirow[b]{3}{*}{ Binding residues } \\
\hline & \multicolumn{3}{|c|}{ Autodock Vina } & \multicolumn{3}{|c|}{ Swissdock } & \\
\hline & Average & Stdev & $\begin{array}{l}\mathrm{CV} \\
(\%)\end{array}$ & Average & Stdev & $\begin{array}{l}\text { CV } \\
(\%)\end{array}$ & \\
\hline Phycoerythrobilin & -7.45 & 0.05 & $0.7 \%$ & -10.35 & 0.0 & 0.0 & $\begin{array}{l}\text { ARG403 TYR449 LEU455 SER494** GLY496* } \\
\text { ASN501* TYR505* }\end{array}$ \\
\hline Phycocyanobilin & -7.25 & 0.15 & 2.1 & -9.355 & 0.025 & 0.3 & SER494** GLY496* GLN498* \\
\hline Dieckol & -8.1 & 0 & 0.0 & -8.375 & 0.045 & 0.5 & $\begin{array}{l}\text { GLU406 LYS417 TYR453* LEU455 GLY496* } \\
\text { THR500* ASN501* TYR505 }\end{array}$ \\
\hline Phycourobilin & -7.1 & 0.0 & 0.0 & -9.285 & 0.425 & 4.6 & $\begin{array}{l}\text { ARG403 LYS417 TYR449 TYR453 SER494** } \\
\text { GLY496** ASN501* TYR505 }\end{array}$ \\
\hline Folic acid & -6.95 & 0.05 & 0.7 & -10.35 & 0 & 0.0 & $\begin{array}{l}\text { ARG403* LEU492** SER494 GLY496** } \\
\text { ASN501* TYR505* }\end{array}$ \\
\hline Pavetannin C1 & -7.3 & 0.3 & 4.1 & -8.52 & 0.14 & 1.6 & LEU492* GLN493 SER494* GLY496* TYR505* \\
\hline Rutin & -7.25 & 0.05 & 0.7 & -8.085 & 0.005 & 0.1 & $\begin{array}{l}\text { ARG403* GLN493** GLY496* ASN501* } \\
\text { TYR505** }\end{array}$ \\
\hline$\beta$-carotene & -7.15 & 0.05 & 0.7 & -8.275 & 0.265 & 3.2 & PHE456 TYR489 PHE490 \\
\hline Astaxanthin & -7.05 & 0.05 & 0.7 & -8.195 & 0.375 & 4.6 & PHE456 ALA475 TYR489 PHE490 \\
\hline
\end{tabular}

Underlined residue reflects unfavorable interactions between themolecule studied and the spike RBD; residues in bold are directlyinvolved in the link between ACE2 and spike RBD. Stars were addedto amino acids involved in one (*) or two (**) H-bonds

CV: Coefficient of variation; Stdev: Standard deviation

Publisher's note Springer Nature remains neutral with regard to jurisdic-

tional claims in published maps and institutional affiliations. 